\title{
PIV study of flow through porous structure using refractive index matching
}

\author{
Richard Häfeli • Marco Altheimer • \\ Denis Butscher · Philipp Rudolf von Rohr
}

Received: 15 November 2013/Revised: 8 February 2014/Accepted: 31 March 2014/Published online: 23 April 2014

(C) Springer-Verlag Berlin Heidelberg 2014

\begin{abstract}
An aqueous solution of sodium iodide and zinc iodide is proposed as a fluid that matches the refractive index of a solid manufactured by rapid prototyping. This enabled optical measurements in single-phase flow through porous structures. Experiments were also done with an organic index-matching fluid (anisole) in porous structures of different dimensions. To compare experiments with different viscosities and dimensions, we employed Reynolds similarity to deduce the scaling laws. One of the target quantities of our investigation was the dissipation rate of turbulent kinetic energy. Different models for the dissipation rate estimation were evaluated by comparing isotropy ratios. As in many other studies also, our experiments were not capable of resolving the velocity field down to the Kolmogorov length scale, and therefore, the dissipation rate has to be considered as underestimated. This is visible in experiments of different relative resolutions. However, being near the Kolmogorov scale allows estimating a reproducible, yet underestimated spatial distribution of dissipation rate inside the porous structure. Based on these results, the $k-\varepsilon$ model was used to estimate the turbulent diffusivity. Comparing it to the dispersion coefficient obtained in the same porous structure, we conclude that even at $R e_{p}=500$ the turbulent diffusivity makes up only a small part of mass transfer in axial direction. The main part is therefore attributed to Taylor dispersion.
\end{abstract}

R. Häfeli $(\square)$ · M. Altheimer · D. Butscher ·

P. Rudolf von Rohr

Institute of Process Engineering, ETH Zürich, Sonneggstrasse 3, 8092 Zürich, Switzerland

e-mail: haefelir@ethz.ch

\section{List of symbols}

\section{Roman symbols}

$C_{\mu} \quad$ Model constant (-)

$D \quad$ Empty pipe diameter (m)

$d_{p} \quad$ Pore diameter $(\mathrm{m})$

$k \quad$ Turbulent kinetic energy $\left(\mathrm{m}^{2} / \mathrm{s}^{2}\right)$

$l_{K} \quad$ Kolmogorov length scale $(\mathrm{m})$

$\hat{l}_{K} \quad$ True Kolmogorov length scale (m)

$n \quad$ Refractive index (-)

$u_{i} \quad$ Velocity in $x_{i}$ direction $(\mathrm{m} / \mathrm{s})$

$\bar{u}_{i} \quad$ Temporal mean velocity in $x_{i}$ direction $(\mathrm{m} / \mathrm{s})$

$u_{i}^{\prime} \quad$ Velocity fluctuation in $x_{i}$ direction $(\mathrm{m} / \mathrm{s})$

$x_{1} \quad$ Main flow axis (m)

$x_{2} \quad$ In-plane axis perpendicular to flow $(\mathrm{m})$

$x_{3} \quad$ Out of plane axis (m)

$\operatorname{Pr}_{T} \quad$ Turbulent Prandtl number (-)

Re Empty pipe Reynolds number (-)

$R e_{p} \quad$ Pore Reynolds number (-)

\section{Greek letters}

$\Delta \quad$ Resolution of PIV measurements (m)

$\varepsilon \quad$ Dissipation rate of turbulent kinetic energy $\left(\mathrm{m}^{2} / \mathrm{s}^{3}\right)$

$\hat{\varepsilon} \quad$ True dissipation rate of turbulent kinetic energy $\left(\mathrm{m}^{2} / \mathrm{s}^{3}\right)$

$\phi \quad$ Scalar quantity

$\lambda \quad$ Scaling factor (-)

$v \quad$ Kinematic viscosity $\left(\mathrm{m}^{2} / \mathrm{s}\right)$

$\Sigma_{\text {eff }} \quad$ Effective diffusivity $\left(\mathrm{m}^{2} / \mathrm{s}\right)$

\section{Abbreviations}

CCD Charge-coupled device

LDV Laser Doppler velocimetry 
LIF Laser-induced fluorescence

P\&ID Piping and instrumentation diagram

PIV Particle image velocimetry

PTV Particle tracking velocimetry

RI Refractive index

\section{Sub- and superscripts}

$o \quad$ Experiment with original dimension

$m \quad$ Model experiment with scaled dimension

max Minimum value

min Maximum value

rms Root mean square

scaled Values scaled to the experiment with salt solution in the large structure

\section{Introduction}

Porous structures find their application as structural material, in heat exchangers, as flame arrestors, as catalyst support and in many other fields like summarized by Banhart (2001). In our research group, a specific geometry of a porous structure was designed for the application as catalyst support in tubular reactors (Hutter et al. 2011). In comparison with a packed bed of catalyst particles, the porous structure reactor has a very high porosity of $78 \%$. This enables high flowrates at comparable low pressure drop. Additionally the advantage compared to a batch reactor with suspended catalyst particles is its improved safety against thermal explosion due to the high heat transfer rate. It is therefore possible to operate at higher concentration, pressure and temperature and use higher amounts of catalyst. For this reason the transition from batch vessels to continuous reactors is seen as a promising approach for process intensification leading to smaller integrated utilities with reduced equipment and operation costs. Due to the increased reaction rate, mass transfer limitations can become important. The transport of mass comprises molecular diffusion but also transport by the motion of the fluid. At low Reynolds numbers the flow is laminar and transport is observed along streamlines. At increased flowrates, however, there can be vortex shedding behind ligaments and even turbulent flow. In order to investigate mass transport, the flow through a porous structure is investigated experimentally by particle image velocimetry. To enable optical measurement techniques, the refractive index (RI) of the liquid and the solid has to be matched. There are two reviews on this subject. Budwig (1994) summarizes the early stage achievements, and Wiederseiner et al. (2011) give a detailed overview of solids and liquids as well as applied measurement techniques. Refractive index matching was often applied to a random packing of particles, a fluidized bed or particle laden flow. In our case, we investigate flow through a porous structure. This structure is reproducibly manufactured by rapid prototyping. The challenge is that our solid has a refractive index of $n=1.519$. This is rather high compared to frequently used RI matching systems. Very often PMMA (polymethylmethacrylate) is used as a solid together with an aqueous salt solutions or an organic liquid with a refractive index of $n=1.49$. Other solid materials such as glass, many transparent plastics such as polyvinylacetate and also silicone rubber have an even lower refractive index. Generally, the choice of liquids is much higher for low RI, whereas for high RI the available liquids are often toxic, rather expensive and have a high viscosity (Hassan and Dominguez-Ontiveros 2008). The liquid, which was found for our application, has a low viscosity and is not toxic nor flammable. On the other hand, it is rather expensive and therefore only applicable in a setup of reasonably small volume.

Measurement techniques applied together with RI matching include PIV, PTV, LDV and LIF. We use PIV to measure the turbulent kinetic energy and the dissipation rate which can be further used to evaluate the eddy diffusivity and length scales of motion. The dissipation rate of turbulent kinetic energy was measured before by PIV in stirred tanks (Sharp and Adrian 2001; Delafosse et al. 2011; Gabriele et al. 2009; Baldi and Yianneskis 2003), in a rectangular channel (Saarenrinne and Piirto 2000), in a zero-mean turbulence box (de Jong et al. 2009) and in ocean bottom boundary layers (Doron et al. 2000). This work extends the spectrum of experiments to flow through porous structures and investigates the scaling of turbulent quantities by employing Reynolds similarity.

An important aspect of porous media fluid dynamics is the identification of flow regimes. Therefore, we briefly review the most important literature on that topic. With increasing Reynolds number, the flow changes from laminar to transitional and turbulent. The transition from laminar to turbulent is usually classified into four flow regimes (Dybbs and Edwards 1984): (1) At very low Reynolds numbers $(R e<1)$, the flow is laminar and dominated by viscous forces. In this regime, the pressure drop is linearly proportional to the flowrate. (2) At higher Reynolds numbers (between 1 and 10), inertial forces start to play a role and the relation between pressure drop and flow rate is no longer linear. The flow is, however, still steady. (3) At a Reynolds number between $\sim 150$ and 300, fluctuation is observed starting with laminar wake oscillations with preferred frequencies. (4) A highly unsteady and chaotic flow regime starts at $R e \sim 300$.

The flow through porous media is frequently described by a pore Reynolds number which is calculated by either the pore diameter (Häfeli et al. 2013), cell diameter (Dukhan and Suleiman 2014), ligament diameter (Zenklusen et al. 
2014), particle diameter (Jolls and Hanratty 1966) or the diameter based on the specific surface area (Horneber et al. 2012). The choice of the characteristic diameter therefore strongly influences the Reynolds number. In our geometry, for example, the cell diameter is 2.8 times larger than the pore diameter. It is clear that choosing the cell diameter instead of the pore diameter as the characteristic length scale leads to much higher Reynolds numbers. This explains why in general it is difficult to compare studies of different authors. So, what should be taken as the characteristic diameter? It depends on the purpose of the investigation. Some porous structures are built up as a network of cylindrical rods. In such a case, the results are frequently compared to studies on flow around single rods (Zenklusen et al. 2014). It is therefore useful to take the rod diameter as the characteristic diameter. For the same reason, the sphere diameter is taken as the characteristic dimension for studies in packed beds of spheres. However, if the goal is to compare flow through porous structures of arbitrary geometry, the choice of the hydraulic diameter as the characteristic diameter could make sense.

\section{Theoretical background}

\subsection{Scalar transport modeling}

The conservation of a scalar quantity $\phi$ can be modeled in a simplified way by an averaged conservation equation (e.g., Pope 2000).

$\frac{\bar{D} \bar{\phi}}{\bar{D} t}=\nabla \cdot\left(\Gamma_{\mathrm{eff}} \cdot \nabla \bar{\phi}\right)$

where $\bar{D} / \bar{D} t$ denotes the mean substantial derivative, $\nabla$ is the gradient operator and $\nabla$. the divergence. The coefficient $\Gamma_{\text {eff }}$ is the effective diffusivity, which is defined as the sum of molecular and turbulent diffisivity

$$
\Gamma_{\text {eff }}=\Gamma+\Gamma_{T}
$$

where $\Gamma$ is the molecular diffusion, and $\Gamma_{T}$ the turbulent diffusion. Usually, the molecular diffusion can be neglected as it is much smaller.

Various models exist that relate the turbulent heat and mass transport to certain characteristics of a turbulent flow. One possibility is the standard $k-\varepsilon$ model developed by Jones and Launder (1972) and slightly modified by Launder and Sharma (1974). This model gives the turbulent diffusivity as

$$
\Gamma_{T}=\frac{v_{T}}{P r_{T}}\left[m^{2} / s\right]
$$

where $v_{T}$ is the turbulent viscosity and $P r_{T}=0.9$ the turbulent Prandtl number. The turbulent viscosity is estimated as
$v_{T}=C_{\mu} k^{2} / \varepsilon$

where $C_{\mu}=0.09$ is one of the five model constants, $k$ is the turbulent kinetic energy, and $\varepsilon$ is the dissipation rate of turbulent kinetic energy. In our case, $k$ and $\varepsilon$ are measured by means of particle image velocimetry (PIV). Combining Eqs. (3) and (4), the turbulent diffusivity can be calculated as:

$$
\Gamma_{T}=\frac{C_{\mu} \cdot k^{2}}{\operatorname{Pr}_{T} \cdot \varepsilon}
$$

\subsection{Turbulent kinetic energy}

The velocity fluctuations used for the determination of the turbulent kinetic energy are obtained by the Reynolds decomposition of the velocity vector:

$u_{i}=\overline{u_{i}}+u_{i}^{\prime}$

where $u_{i}(\mathrm{~m} / \mathrm{s})$ : measured velocity, $\overline{u_{i}}(\mathrm{~m} / \mathrm{s})$ : mean velocity and $u_{i}^{\prime}(\mathrm{m} / \mathrm{s})$ : velocity fluctuation in direction $x_{i}$.

From the velocity fluctuation in all three dimensions, the turbulent kinetic energy is calculated:

$k=\frac{1}{2} \cdot \overline{u_{i}^{\prime} u_{i}^{\prime}}=\frac{1}{2} \cdot\left(\overline{u_{1}^{\prime} u_{1}^{\prime}}+\overline{u_{2}^{\prime} u_{2}^{\prime}}+\overline{u_{3}^{\prime} u_{3}^{\prime}}\right)$

With 2D PIV measurements, only two components of the velocity field are measured. We therefore have to make assumptions to calculate the turbulent kinetic energy. A discussion of this can be found in Sect. 4.1.

\subsection{Dissipation rate}

The dissipation rate for a fluid with viscosity $v$ is defined as

$\varepsilon=\frac{1}{2} v \overline{\left(\frac{\partial u_{i}^{\prime}}{\partial x_{j}}+\frac{\partial u_{j}^{\prime}}{\partial x_{i}}\right)^{2}}$

This equation can be expanded leading to:

$$
\varepsilon=v\left\{\begin{array}{l}
\left.2 \overline{\left(\frac{\partial u_{1}^{\prime}}{\partial x_{1}}\right)^{2}}+\overline{\left(\frac{\partial u_{2}^{\prime}}{\partial x_{2}}\right)^{2}}+\overline{\left(\frac{\partial u_{3}^{\prime}}{\partial x_{3}}\right)^{2}}\right) \\
+\overline{\left(\frac{\partial u_{1}^{\prime}}{\partial x_{2}}\right)^{2}}+\overline{\left(\frac{\partial u_{2}^{\prime}}{\partial x_{1}}\right)^{2}}+\overline{\left(\frac{\partial u_{1}^{\prime}}{\partial x_{3}}\right)^{2}}+\overline{\left(\frac{\partial u_{3}^{\prime}}{\partial x_{1}}\right)^{2}} \\
+\overline{\left(\frac{\partial u_{2}^{\prime}}{\partial x_{3}}\right)^{2}}+\overline{\left(\frac{\partial u_{3}^{\prime}}{\partial x_{2}}\right)^{2}} \\
+2\left(\frac{\partial u_{1}^{\prime} \partial u_{2}^{\prime}}{\partial x_{2}} \frac{\overline{\partial u_{1}^{\prime}} \frac{\partial u_{3}^{\prime}}{\partial x_{3}}}{\partial x_{1}}+\frac{\partial u_{2}^{\prime} \partial \frac{\partial u_{3}^{\prime}}{\partial x_{3}}}{\partial x_{2}}\right)
\end{array}\right\}
$$

The terms containing $u_{3}$ or $x_{3}$ are not measured by 2D PIV. Instead, the unknown terms need to be estimated from the known terms by proper assumptions. If homogeneous and 
isotropic turbulence is assumed, the dissipation rate can be simply calculated from one gradient (de Jong et al. 2009):

$$
\varepsilon=15 v \overline{\left(\frac{\partial u_{1}^{\prime}}{\partial x_{1}}\right)^{2}}
$$

There are various models available which lie between Eqs. (9) and (10) depending on the assumptions made. Depending on the flow situation, axisymmetry (George and Hussein 1991) or isotropy (Sharp and Adrian 2001) can be assumed. Further, the mixed derivative moments can be transformed using the incompressible continuity equation like introduced by George and Hussein (1991). Finally, some authors used the continuity equation to estimate the cross-product gradients (Doron et al. 2000).

Delafosse et al. (2011) investigated the flow in a stirred tank by PIV. They observed that the isotropy ratios, defined as ratios between squared gradients and cross-product gradients, are not all equal to unity. Based on that, a formula for $\varepsilon$ was proposed:

$$
\begin{aligned}
\varepsilon & =v\left\{\overline{\left\{\left(\frac{\partial u_{1}^{\prime}}{\partial x_{1}}\right)^{2}\right.}+3 \overline{\left(\frac{\partial u_{2}^{\prime}}{\partial x_{2}}\right)^{2}}\right. \\
& \left.+3 \overline{\left(\frac{\partial u_{1}^{\prime}}{\partial x_{2}}\right)^{2}}+3 \overline{\left(\frac{\partial u_{2}^{\prime}}{\partial x_{1}}\right)^{2}}+6 \overline{\left(\frac{\partial u_{1}^{\prime}}{\partial x_{2}} \frac{\partial u_{2}^{\prime}}{\partial x_{1}}\right)}\right\}
\end{aligned}
$$

Much more sensitive than the choice of the right model is the requirement to have an appropriate spatial resolution of the measurements. As the dissipation occurs at the smallest scales of motion, i.e., at the Kolmogorov scale, the measurement should resolve even these motions. It was observed in several studies that the error can be high when the resolution is insufficient. For example, Saarenrinne et al. (2001) observed that if the resolution is around $2 l_{K}$, the estimated dissipation rate is $90 \%$ of the true value. If the resolution is $9 l_{K}$, the measured value corresponds to $65 \%$ of the true value. Baldi and Yianneskis (2003) observed that doubling (improving) the spatial resolution leads to a $250 \%$ increase in the dissipation rate. Delafosse et al. (2011) observed that doubling the spatial resolution increased the estimated dissipation rate by $220 \%$.

\subsection{Scaling laws}

Scaling laws are applied in order to compare experiments with anisole and the salt solution as well as experiments in ducts of 7 and $20 \mathrm{~mm}$ diameter. In wind tunnel experiments, it is common to adjust size, viscosity and velocity in a way that makes the experiment realizable in a wind tunnel but keeping the Reynolds number constant:

$$
\frac{u_{o} D_{o}}{v_{o}}=R e_{o}=R e_{m}=\frac{u_{m} D_{m}}{v_{m}}
$$

where $o$ indicates the original experiment with original size and viscosity and $m$ the experiment with the model settings. When the size of the object under investigation is changed, this has to be done uniformly in all three dimension, i.e., geometric similarity has to be given. If two objects are geometrically similar and experiments are done at equal Reynolds number, then kinematic similarity holds. This means that ratios between corresponding velocities are equal in the original and in the model experiment:

$$
\left.\frac{u_{r m s}^{\prime}}{\bar{u}}\right|_{o}=\left.\frac{u_{r m s}^{\prime}}{\bar{u}}\right|_{m}
$$

With the help of this, the ratio of the turbulent kinetic energy can be calculated from the ratio of a mean velocity:

$$
\frac{k_{m}}{k_{o}}=\frac{\left.\overline{u^{\prime} u^{\prime}}\right|_{m}}{\left.\overline{u^{\prime} u^{\prime}}\right|_{o}}=\frac{\overline{u_{m} u_{m}}}{\overline{u_{o} u_{o}}}
$$

In our experiments, we set the diameter and the viscosity and then adjust the velocity of the model experiment to ensure Reynolds similarity. In a series of measurements, we then vary the velocity. We therefore like to have a scaling law which bases on a priori known and constant variables, i.e., $v$ and $D$. Combining Eqs. (12) and (15) leads to:

$$
\frac{k_{m}}{k_{o}}=\frac{v_{m}^{2} D_{o}^{2}}{v_{o}^{2} D_{m}^{2}}
$$

or

$$
k \propto \frac{v^{2}}{D^{2}}
$$

The same procedure is applied to scale the values of the dissipation rate:

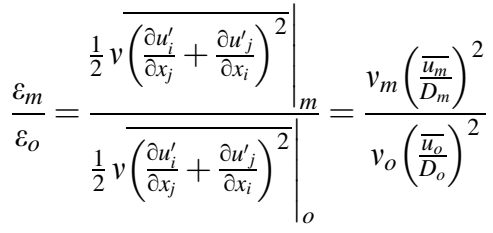

If we again replace $u$ by employing that $R e_{o}=R e_{m}$, we obtain a scaling law which is only depending on a priori known variables:

$\varepsilon \propto \frac{v^{3}}{D^{4}}$.

Finally, the scaling law of the turbulent diffusivity is:

$\frac{\Gamma_{T, m}}{\Gamma_{T, o}}=\frac{k_{m}^{2} / \varepsilon_{m}}{k_{o}^{2} / \varepsilon_{o}}=\frac{v_{m}}{v_{o}}$

or in other words

$\Gamma_{T} \propto v$. 
Table 1 Scaling factors for the experiments with anisole and in the small structure

\begin{tabular}{llll}
\hline & $k$ & $\varepsilon$ & $\Gamma_{T}$ \\
\hline Large structure with saltwater (reference) & 1 & 1 & 1 \\
Large structure with anisole & 0.4876 & 0.329 & 0.69 \\
Small structure with saltwater & 8.163 & 66.64 & 1 \\
\hline
\end{tabular}

The experiment with salt in the large structure remains unscaled

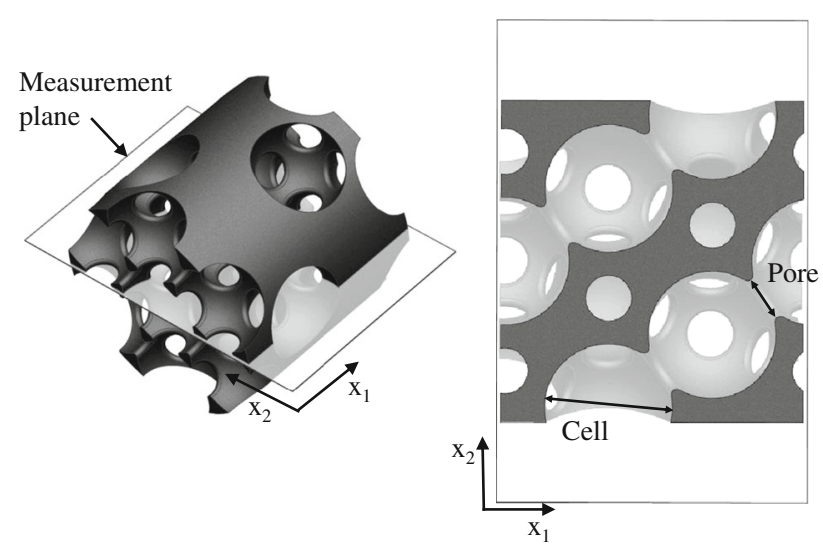

Fig. 1 One periodic unit of the porous structure. Fluid flow in axial direction $x_{1}$. Left auxiliary view, right cut at measurement plane

We define that the original experiment is the one with salt solution in a diameter of $20 \mathrm{~mm}$ and the other experiments are model experiments which are scaled to the values of the original. Table 1 shows the numerical values of the scaling factors.

\section{Experimental}

\subsection{Investigated structures}

The investigated structures are made of Somos ${ }^{\circledR}$ WaterShed XC 11122, in the following called WaterShed. It is a transparent material developed for the manufacturing with stereolithography. The porous structure is designed as the negative of tetrahedrally arranged overlapping spheres. Figure 1 shows an auxiliary view of one periodic unit of the porous structure (left) and a top view on the measurement plane (right). The large void spaces inside the structure are in the following referred to as cells. The openings between two adjacent cells will be called pores. Every cell is connected to twelve other cells. The fluid mainly enters a cell through its three upstream neighbors and mainly leaves through the three downstream neighbors. The remaining six neighbors are found perpendicular to the main flow axis, and therefore, there is only minor flow to
Table 2 Characteristic dimensions of the two porous structures

\begin{tabular}{lll}
\hline & Small structure & Large structure \\
\hline Empty pipe diameter $D(\mathrm{~mm})$ & 7 & 20 \\
Cell diameter $(\mathrm{mm})$ & 2.78 & 7.94 \\
Cell distance $(\mathrm{mm})$ & 2.75 & 7.86 \\
Rounding radius $(\mathrm{mm})$ & 0.12 & 0.34 \\
Pore diameter $d_{p}(\mathrm{~mm})$ & 1.01 & 2.88 \\
Porosity $(\%)$ & 78.7 & 78.7 \\
Hydraulic diameter $(\mathrm{mm})$ & 2.38 & 6.79 \\
\hline
\end{tabular}

these cells. The measurements are made in a plane through the center of the tube. From the total of twelve pores per cell, only two are in the measurement plane. As a consequence, there is only one entrance and one exit visible in the PIV images.

The original size porous structure fits inside a tube of diameter $20 \mathrm{~mm}$. A downscaled model is manufactured with a scaling factor of $\lambda=7 / 20=0.35$. The parameters of these two structures are given in Table 2. It was found that in the small-scale porous structure, there is a significant deviation between CAD drawings and manufactured structure. This is due to the layer thickness which is $0.05 \mathrm{~mm}$ in the manufacturing unit of our supplier (von Allmen AG, Switzerland). Therefore, the exact geometry of the small structure was measured by X-ray tomography (Hutter et al. 2011). This measurements allowed to determine the exact geometry with a resolution of $5 \mu \mathrm{m}$. The values given in Table 2 for the small structure represent the dimensions fitted to the model from the X-ray scan. In the large structure, the relative deviation of the designed dimension to the manufactured dimension is neglected. This is motivated by the high resolution of the manufacturing process in relation to the size of the porous structure.

For the measurement of the refractive index, an Abbe refractometer from CarlZeiss $\mathrm{AG}$ was used. As the refractive index changes with temperature, the measurement instrument was connected to a thermostat. There are two measurement principles possible which both base on the measurement of the angle of total reflectance. The angle can be measured either in reflected light or in transmitted light. For the liquids, the measurements were done in transmitted light, whereas for the solid the measurement was done in reflected light. The refractive index as a function of temperature is shown in Fig. 2. According to the datasheet of the manufacturer, the refractive index of the WaterShed is between 1.512 and 1.515 (Somos 2012). We observed a RI of 1.519. The reason for this deviation could be a result of the difference in manufacturing procedure or in measurement inaccuracy. One problem observed during the experiments was that WaterShed absorbs water. After 1 month in still water, a plate of 


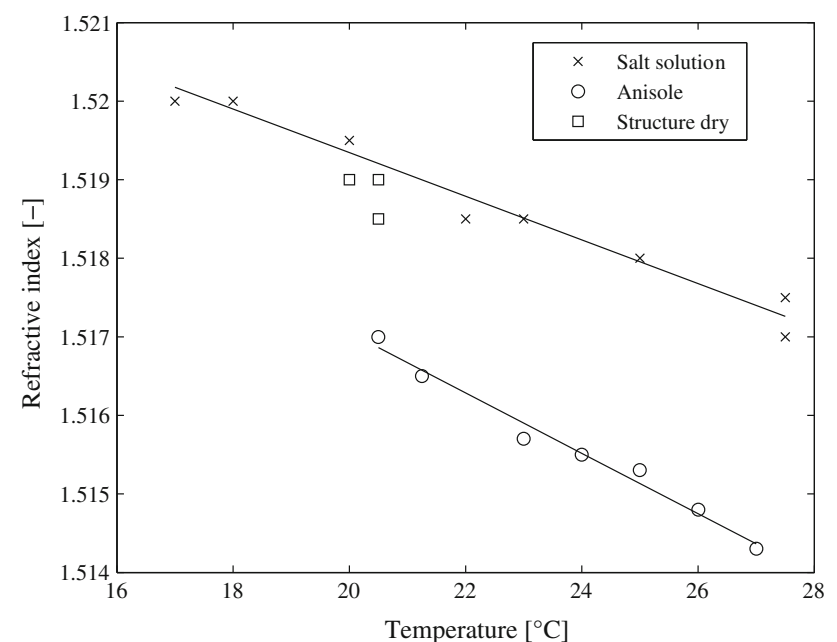

Fig. 2 Refractive index as a function of temperature for the two fluids and for the solid phase

WaterShed of $4 \mathrm{~mm}$ thickness is $1 \%$ heavier due to absorbed water. It was found that this significantly increases the refractive index. While the dry structure has a refractive index of $n=1.519$, a structure that absorbed water reaches a refractive index above $n=1.530$. In order to prevent RI changes, WaterShed was cleaned every day with distilled water and then flushed with dry compressed air in order to allow drying and desorption. By control of the weight of the structure, it was checked that no water was absorbed over time.

For the measurements with anisole, the structure had to be coated because the solid is dissolved by anisole. For that, we used DS nuvovern (Mäder Aqualack AG, Switzerland), a varnish based on a polyurethane resin. The small structure was not suitable for the coating procedure due to surface forces which made it difficult to let the varnish flow through the structure by gravity. We therefore decided to do measurements with anisole only in the large structure. For the measurements with salt solution, no coating is required and therefore the experiments could be done also in the small structure. For an estimation of the influence of the coating, the experiments in the large structure with the salt solution were done twice-once with coating and once without.

\subsection{Fluids}

Two fluids were used in the experiment. The first is anisole, an organic liquid which was found by Butscher et al. (2012) to be suitable as fluid to match the refractive index of WaterShed. The second fluid is an aqueous solution of sodium iodide and zinc iodide. An overview of the properties is given in Table 3. The presented data are from own measurements. The density was measured with the coriflow
Table 3 Properties of the two fluids (own measurements)

\begin{tabular}{lll}
\hline & Anisole & Salt solution \\
\hline Density $(\mathrm{g} / \mathrm{l})$ & 990 & 1994 \\
Viscosity $\left(\mathrm{m}^{2} / \mathrm{s}\right)$ & $1.56 \mathrm{E}-6$ & $2.26 \mathrm{E}-6$ \\
Refractive index @ $20^{\circ} \mathrm{C}(-)$ & 1.517 & 1.5195 \\
Price per liter for $11(\mathrm{USD})$ & $\approx 90$ & $\approx 900$ \\
Price per liter for 101 (USD) & $\approx 70$ & $\approx 400$ \\
\hline
\end{tabular}

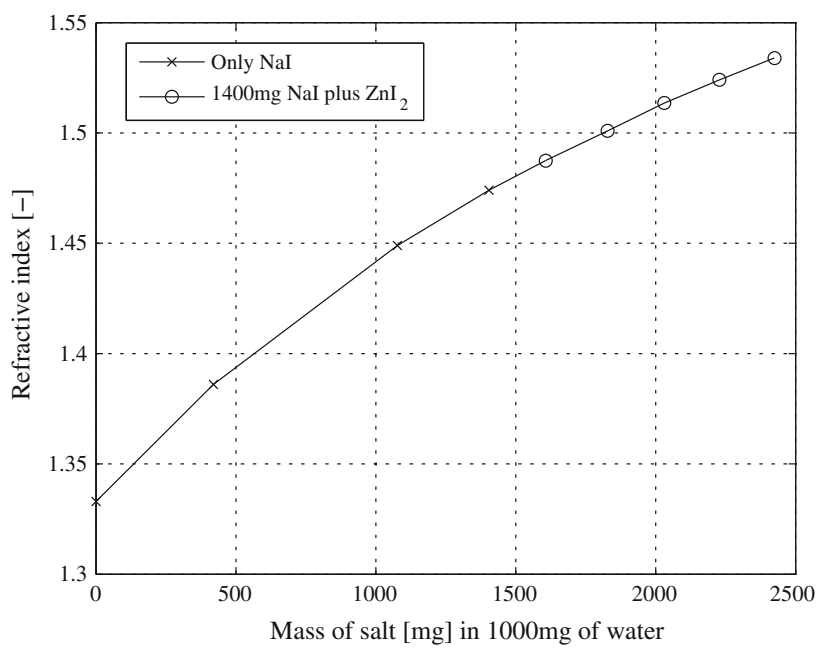

Fig. 3 Refractive index as a function of the composition of the salt solution

massflow meter from Bronckhorst (M55-AGD-22-O-S). For the viscosity measurement, a viscosimeter VT550 from Haake was used. The question might occur why a mixture of two salts is used instead of only one. The reason is that with sodium iodide alone the achievable refractive index near the solubility limit is $n=1.5$ (Budwig 1994). At increased temperatures, the solubility is higher and theoretically a refractive index of $n=1.519$ could be reached. This would however require tempering the whole setup at an increased temperature. On the other hand, a solution of only zinc iodide can reach a refractive index of up to $n=$ 1.62 due to its very high solubility in water (Budwig 1994). However, such a solution would lead to a liquid of high viscosity. Further, the price of zinc iodide is rather high. Therefore, we propose to use a mixture of both fluids: sodium iodide as a cheap material to increase the refractive index and zinc iodide to further increase and match the refractive index to the one of the solid. The refractive index as a function of the composition is shown in Fig. 3. Starting with $1,000 \mathrm{mg}$ of pure water with a refractive index of $n=1.33$, the addition of $\mathrm{NaI}$ increases the refractive index and reaches 1.47 when $1,400 \mathrm{mg}$ is added. To further increase the refractive index zinc iodide is added. It was found that $730 \mathrm{mg}$ of zinc iodide is sufficient 


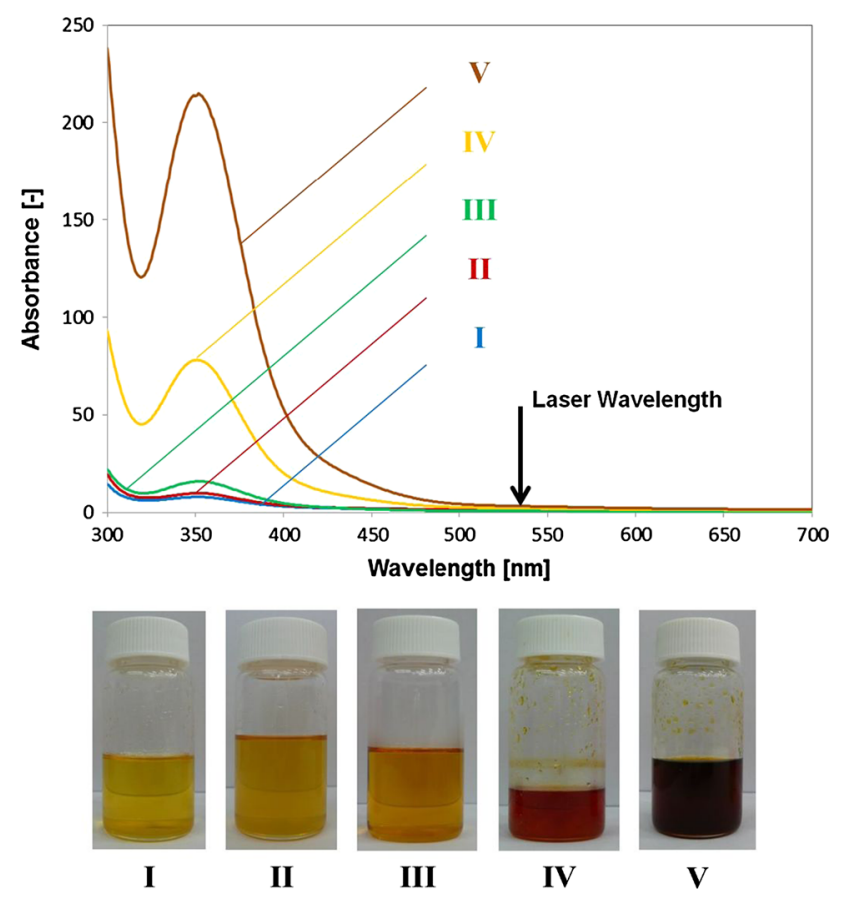

Fig. 4 Light absorption spectrogram of salt solutions with different extent of oxidation

to reach $n=1.519$. The relative amount of the two salts could also be different from our proposal. A higher amount of $\mathrm{NaI}$ could further decrease the price as well as viscosity; however, the solubility limit would soon be reached.

The new fluid has several advantages over anisole. The WaterShed structure was found to be chemically stable in the salt solution. This is the main advantage over anisole which dissolves an uncoated structure rapidly. Even with the protective coating, the structure decomposes after some time. Further, the refractive index could be matched very accurately compared to anisole which is a pure substance. The disadvantage of the salt solution is its discoloration over time. In contact with oxygen, the salt solution changes its color from transparent to yellow (Fig. 4). As we want to do optical measurements with a laser of wavelength $532 \mathrm{~nm}$, we checked the absorbtion spectrum of the yellow salt solution. We found that the maximum absorbance is at lower wavelengths, i.e., around $350 \mathrm{~nm}$ (blue light is absorbed; therefore, the solution appears yellow). At the wavelength of the laser, the absorbance was negligible. This ensures that measurements can be made even if the solution gets yellow. The yellow color is attributed to the formation of iodine by oxidation of zinc. In small samples, this process was successfully prevented by covering the liquid with nitrogen. Even though we did not flush the storage vessel with nitrogen, the discoloration was very slow. This was probably because of the low surface to volume ratio. Therefore, we could work with the same liquid for a very long time, i.e., for more than one year. If the discoloration occurs and an isolation from oxygen is not feasible, it could be possible to use sodium thiosulfate $\left(\mathrm{Na}_{2} \mathrm{~S}_{2} \mathrm{O}_{3}\right)$ to reduce the iodine to iodide (Narrow et al. 2000). Important to note is that the effect on the physical properties of the fluid is very small. Neither density, viscosity nor RI changed by discoloration. We conclude from this observation that the amount of substance that was oxidized must be very small.

\subsection{The setup}

The P\&ID of the setup is shown in Fig. 5. The fluid is sucked by a gear pump (VGS200 from VerderCatag) from the working fluid tank. It then flows through a coriflow massflow meter (Bronckhorst M55-AGD-22-O-S) which has an accuracy of $0.2 \%$. To ensure constant physical properties, especially refractive index and viscosity, the fluid passes a thermostat which tempers the fluid to $20^{\circ} \mathrm{C}$. To ensure fully developed flow, the fluid then enters an empty pipe of $1 \mathrm{~m}$ length and equal diameter as the measurement section. After passing the measurement section, the liquid is recycled to the tank.

\subsection{Measurement equipment}

A plane in the porous structure is illuminated by a doublepulsed Nd:YAG laser (Litron Nano L 135-15). The images are recorded by a CCD camera with a resolution of $1,600 \times$ 1,200 pixels and 14-bit dynamic range (PCO1600). A BNC575 timing unit is used for the synchronization. The software for control and evaluation of measurements is Dantec DynamicStudio v3.31. Different seeding particles were evaluated. It was found that glass beads and polyamide particles cannot be dispersed in the saltwater. Due to their low density, they float on the surface of the liquid. We therefore choose particles of higher density and found aluminum oxide to be suitable. The aluminum oxide seeding particles have a mean diameter of $2 \mu \mathrm{m}$. This is rather small compared to other PIV studies in liquid flows (Melling 1997). An advantage of small seeding particles is their better flow tracking capability. An other rule for the selection of seeding particle size is that they should cover more than one pixel on the image, preferably around 2.5 pixels in order to prevent peak locking. Even though the pixel size on the image is $8 \mu \mathrm{m}$, the scattered light from $2 \mu \mathrm{m}$ particles illuminates an area of roughly 2.5 pixels. It is therefore considered as a suitable particle size both from flow tracking capability and quality of images.

The sample size for experimental work must be known a priori. Too small sample size results in large statistical uncertainty. With too large sample sizes, time and effort is wasted without increasing the accuracy. In order to find the 
Fig. 5 Scheme of the setup

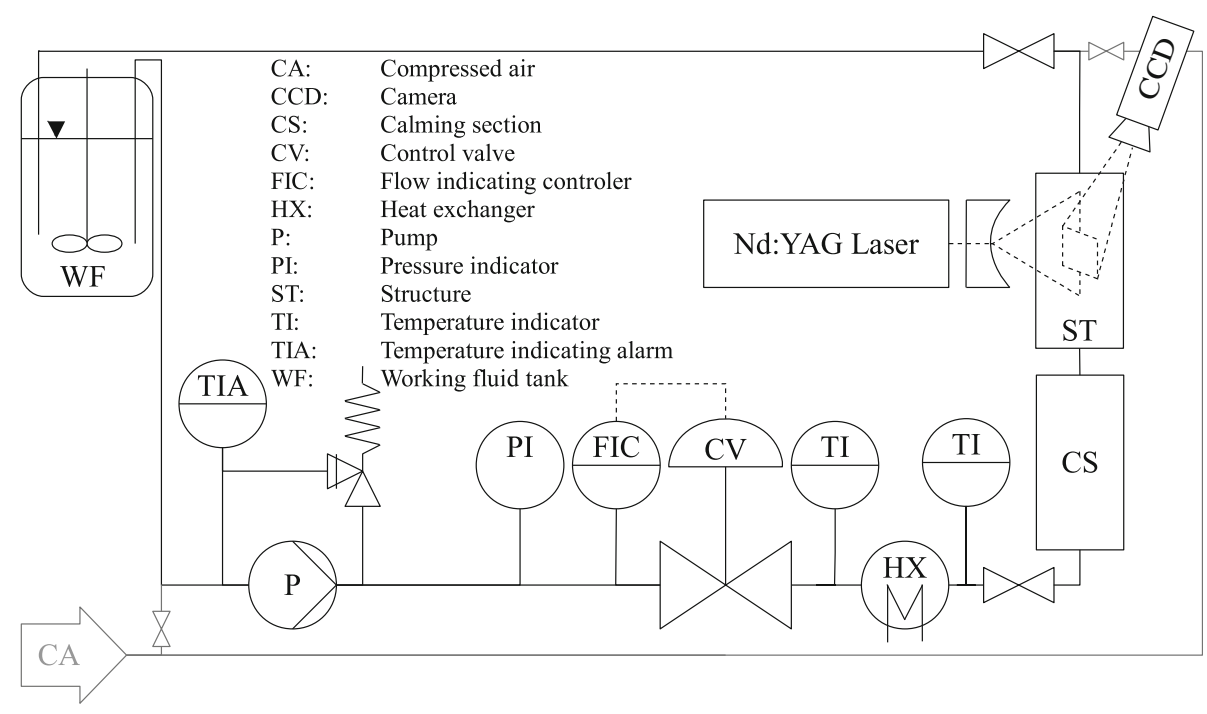

appropriate sample size delivering a statistically meaningful result, we applied bootstrapping. Bootstrapping is a resampling technique that allows to estimate statistical quantities from a set of measurements (Efron 1979). For this, a series was recorded consisting of 10,000 measurements. Then, a sample of $n$ values is drawn out of these 10,000 . From these $n$ values, the average is calculated. Repeating this procedure $B$ times, the standard deviation of the $B$ mean values can be calculated. It was found that for a sample size $n>500$, the statistical deviation of the result (turbulent kinetic energy) does not decrease significantly. With a sample size of 500, the statistical uncertainty is below $0.5 \%$ and no time or effort is wasted. The same procedure for the dissipation rate suggests that for sample size $n=500$, the statistical uncertainty is below $5.2 \%$.

For the evaluation of the images, the mean image is calculated from a series of measurements. The mean image is then subtracted from each image in order to eliminate perturbations from the background. In general, not all tracer particles have the same brightness due to differences in surface properties. To ensure that all particles have the same weight in the cross-correlation, the image could be converted to black-white. This, however, imposes problems in sub-pixel interpolation and leads to peak locking. The alternative is to clamp the image such that bright and dark particles have the same weight. Both background subtraction and clamping are done by a built-in function in the commercial software DynamicStudio from Dantec. Figure 6 shows an image after background subtraction and clamping. The interrogation area size for the cross-correlation was $64 \times 64$ pixel for the measurements in the $20 \mathrm{~mm}$ structure and $32 \times 32$ pixel for the measurements in the $7 \mathrm{~mm}$ structure. In any case, a $50 \%$ overlap of

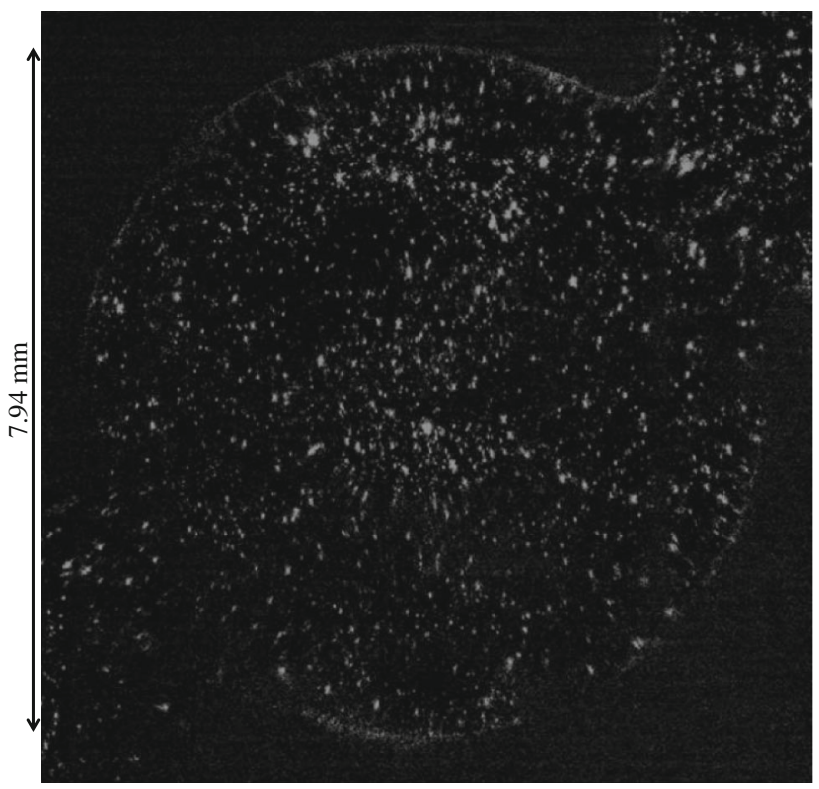

Fig. 6 Image of PIV particles inside a cell of the porous structure after subtraction of mean image and gray value clamping. The image is of a measurement with the large diameter, i.e., with a cell size of $7.94 \mathrm{~mm}$

interrogation areas was used. With these settings, the resolutions were $\Delta_{20 \mathrm{~mm}}=268 \mu \mathrm{m}$ and $\Delta_{7 \mathrm{~mm}}=152 \mu \mathrm{m}$. The resulting velocity vector field had to be postprocessed to remove spurious vectors. This is done by a moving average validation. The procedure compares each vector to the vectors in its surrounding, in our case a region of $3 \times 3$ vectors. If the deviation exceeds an acceptance threshold, the vector is replaced by the average of its surrounding vectors as a reasonable estimate of the true velocity. 


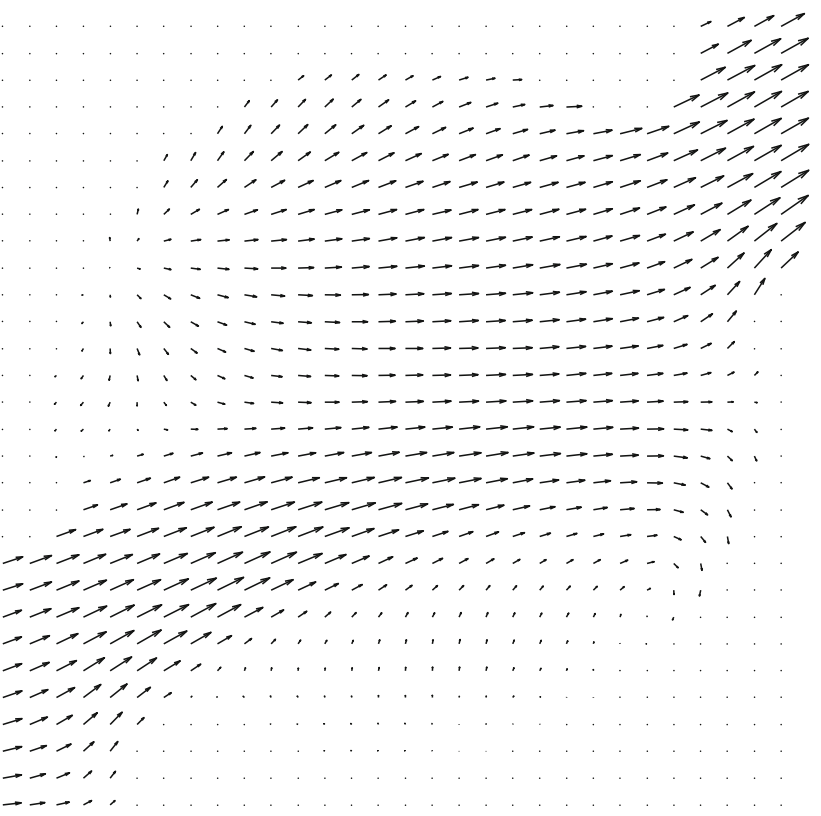

Fig. 7 Mean velocity of an experiment with salt solution in the uncoated large structure at $R e_{p}=457$

\section{Results and discussion}

We first discuss the results on turbulent kinetic energy and dissipation rate of turbulent kinetic energy. After the evaluation of the necessary assumptions, we show local distributions and compare the results by means of Reynolds similarity. In the end, we then discuss scalar transport mechanisms in flow through porous structures by analyzing the turbulent diffusivity.

\subsection{Turbulent kinetic energy}

All following experiments are made in the tenth periodic unit from the entrance to the porous structure. At this position, we can assume fully developed flow (Butscher et al. 2012). The measurements are done with salt solution and anisole in the large structure and with salt solution in the small structure. Figure 7 shows the mean velocity field for the reference measurement with salt solution in the large structure. Only one of three inlets and outlets is visible in the measurement plane. Part of the fluid enters through the pore on the left and then leaves the cell through the two pores on the lower right side (not visible in the measurement plane). This means that the fluid entering through the lower left pore is leaving the plane of measurement. Similarly, the fluid on the upper half of the cell enters through the two pores on the upper left (not in the measurement plane) and leaves through the pore on the upper right. As a consequence of this, there is a horizontal line through the center of the pore through which no fluid flows in average.

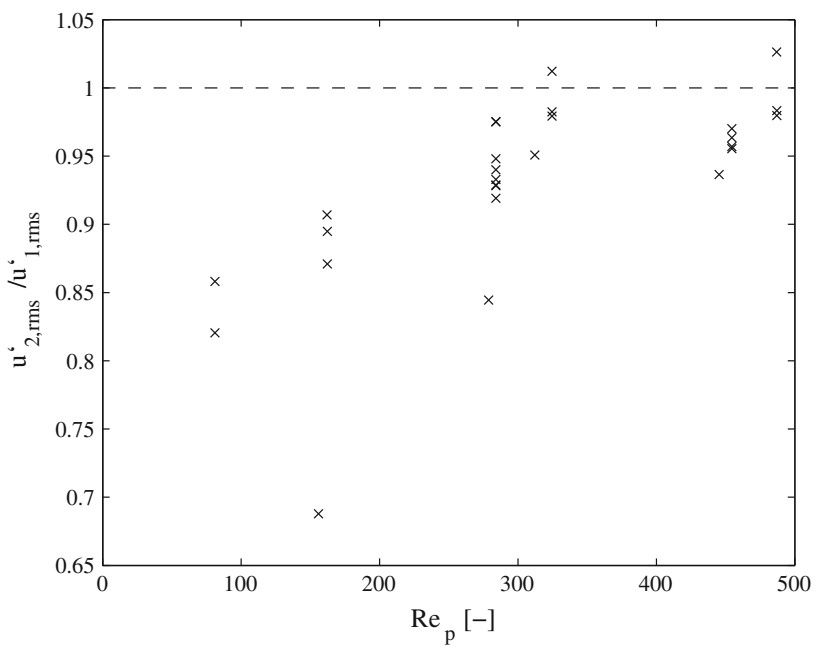

Fig. 8 Ratio between root-mean-square velocity fluctuation in $x_{1}$ and $x_{2}$ direction

As only two components of the velocity fluctuation are measured by PIV, the third component has to be estimated from the first two. Comparing the two components measured by PIV, it was observed that the ratios between velocity fluctuations in $x_{1}$ and $x_{2}$ directions are not equal, especially at low flowrates (Fig. 8). Whereas the turbulence can be considered isotropic at high flowrates, at low flowrates the fluctuations perpendicular to the main flow axis are more than $10 \%$ lower than in the main flow direction. It was therefore decided to calculate the turbulent kinetic energy by assuming axisymmetric flow, i.e., the two components perpendicular to the main flow direction are considered equal:

$k=\frac{1}{2} \cdot\left(\overline{u_{1}^{\prime} u_{1}^{\prime}}+2 \cdot \overline{u_{2}^{\prime} u_{2}^{\prime}}\right)$

In Fig. 9, the profile of turbulent kinetic energy within a cell of the porous structure is shown. The turbulent kinetic energy is highest at the boundary of the jet. On the other hand, the turbulent kinetic energy is very low on the upper half of the pore. What is also observed is that in the region of the outlet the turbulent kinetic energy is underestimated and periodicity is not given. This was observed also with anisole and in the small structure. We therefore have to attribute this to a measurement error, probably stemming from refraction in the region of the pores. The reason is that the curvature of the solid is highest in the region of the pore. Further, the light is passing the phase boundary at a very low incident angle. As the refractive index matching is never perfect, this will always lead to some optical distortions which could lead to the lower value of $k$ in the region of the outlet. In the center of the cell, however, we observed very good agreement between the measurements and a corresponding large-eddy simulation (Hutter et al. 2011). 

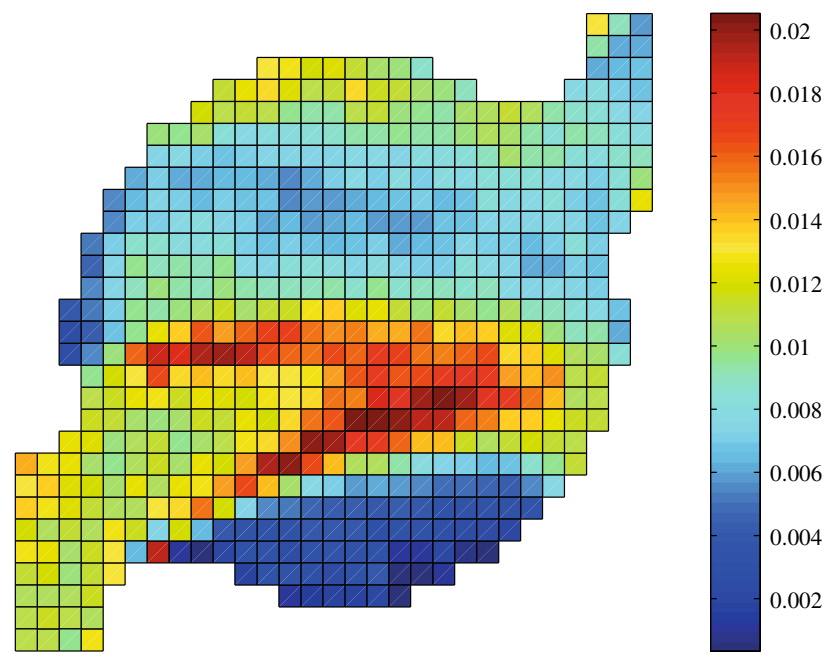

Fig. 9 Turbulent kinetic energy field of an experiment with salt solution in the uncoated large structure at $R e_{p}=457$

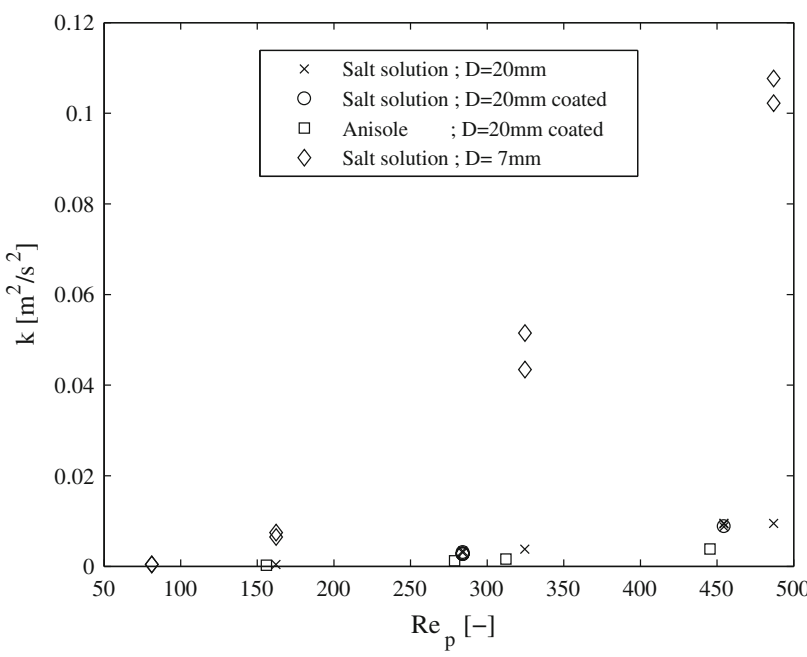

Fig. 10 Turbulent kinetic energy as a function of Reynolds number for different fluid viscosities and structure dimensions without scaling

From the profile of turbulent kinetic energy, the spatial mean value is calculated. The result is shown in Fig. 10. Like expected the turbulence intensity increases with increasing pore Reynolds number. What can be clearly seen is that for a given Reynolds number the turbulent kinetic energy is highest in the measurement in the small structure. The reason is that the characteristic dimension of this structure is a factor of 2.857 smaller. We therefore scale the turbulent kinetic energy with the scaling laws given in Sect. 2.4. The scaled results are given in Fig. 11. A good agreement is found between the measurements. Generally, the turbulent kinetic energy can be measured with a high accuracy by PIV because the turbulent kinetic energy is mainly contained in the big (and therefore resolved) scales. The small-scale motion, which may be

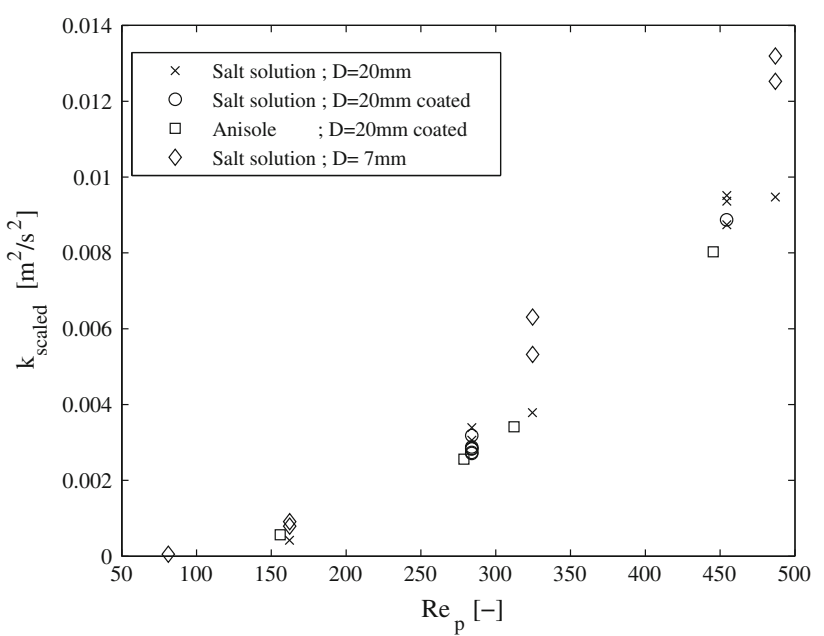

Fig. 11 Scaled turbulent kinetic energy

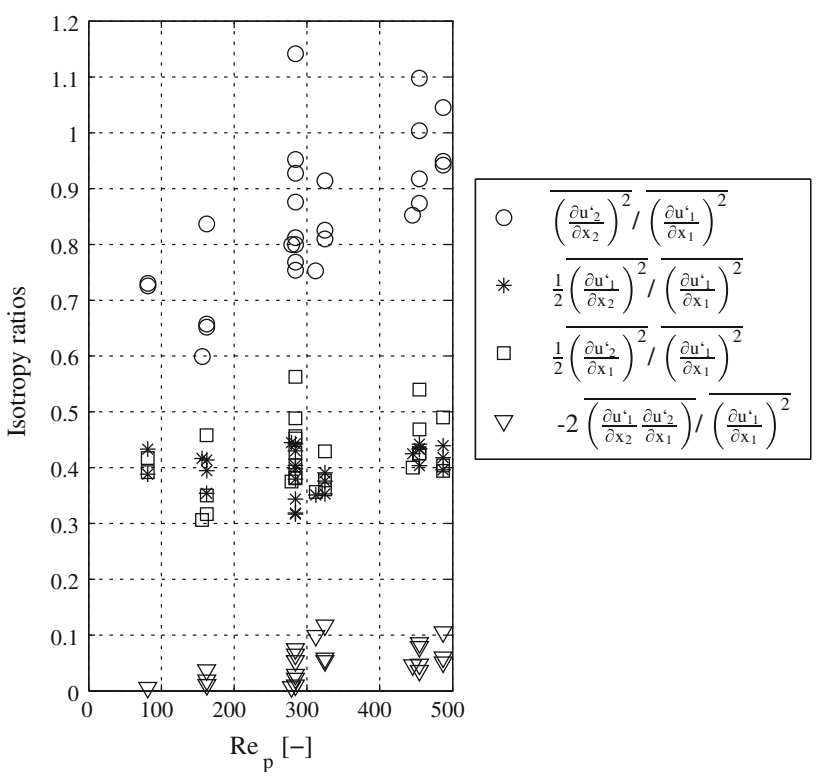

Fig. 12 Ratio between squared gradients and cross-product gradients in $x_{1}$ and $x_{2}$ direction as a function of the pore Reynolds number defined with the interstitial velocity and the pore diameter

unresolved in the PIV measurements, does not contain high kinetic energy, and therefore, their negligence does not impose large errors.

\subsection{Dissipation rate of turbulent kinetic energy}

Next, we evaluated the dissipation rate from the PIV vector fields. First, the isotropy ratios based on Delafosse et al. (2011) were calculated. Figure 12 shows the mean isotropy ratio of every experiment as a function of the pore Reynolds number. It can be seen that only one ratio is $\approx 1$, whereas the others are around $\approx 0.5$ and $\approx 0.1$. This 


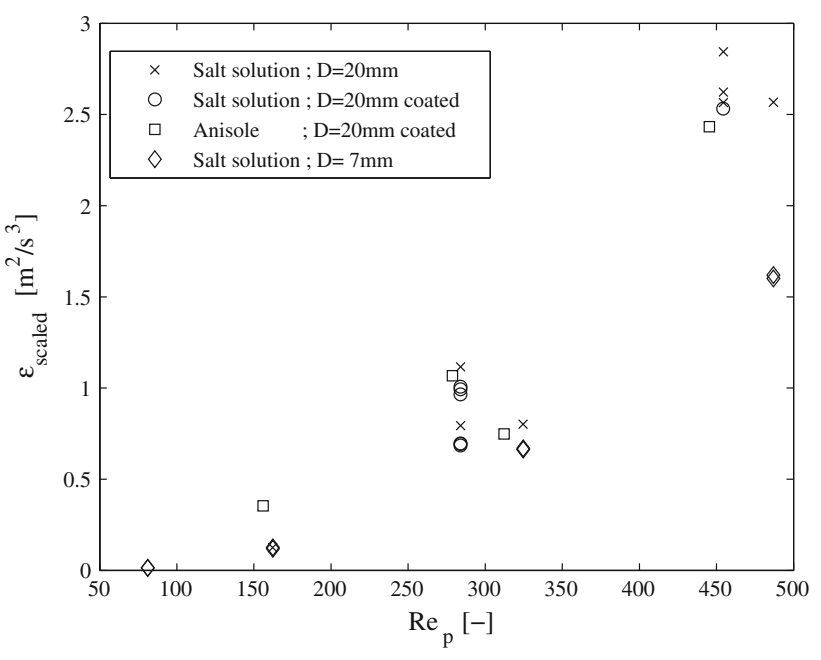

Fig. 13 Scaled dissipation rate as a function of pore Reynolds number

observation is in very good agreement to the work by Delafosse et al. (2011) who investigated the flow in a stirred tank. Therefore, their dissipation rate model seems appropriate also for our case.

If the dissipation rate is plotted against the pore Reynolds number without scaling, we observe the highest values in the measurements in the small structure. The scaling laws introduced in Sect. 2.4 are used to compare the results (Fig. 13). We find that the results have a reasonably small standard deviation for measurements of dissipation rate. In comparison with the measurement of $k$, the deviation between the measurements is higher. What stands out is that the values for the small structure $(D=7 \mathrm{~mm})$ are considerably lower than the others. The reason for this is that the relative resolution in these measurements is worse. It is well known that the dissipation rate is a quantity which cannot be measured accurately with PIV because of the high demands on the spatial resolution (Xu and Chen 2013). As dissipation occurs at the smallest scales of motion, a measurement should resolve these scales. In our case, the resolution $\Delta$ was larger than the Kolmogorov length scale, which was calculated as:

$l_{K}=\left(\frac{v^{3}}{\varepsilon}\right)^{1 / 4}$

To simplify the discussion, we restrict ourselves to the spatially averaged value of the experiment with saltwater in the large structure. We find that $l_{K, \max }=98 \mu \mathrm{m}$ at the lowest Reynolds number and $l_{K, \text { min }}=45 \mu \mathrm{m}$ at the highest Reynolds number. The resolution of the PIV measurements in the same experiment was $\Delta=268 \mu \mathrm{m}$. Therefore, in relation to the Kolmogorov scales, our resolution is a factor of 2.7-6 lower. When the resolution is insufficient, the

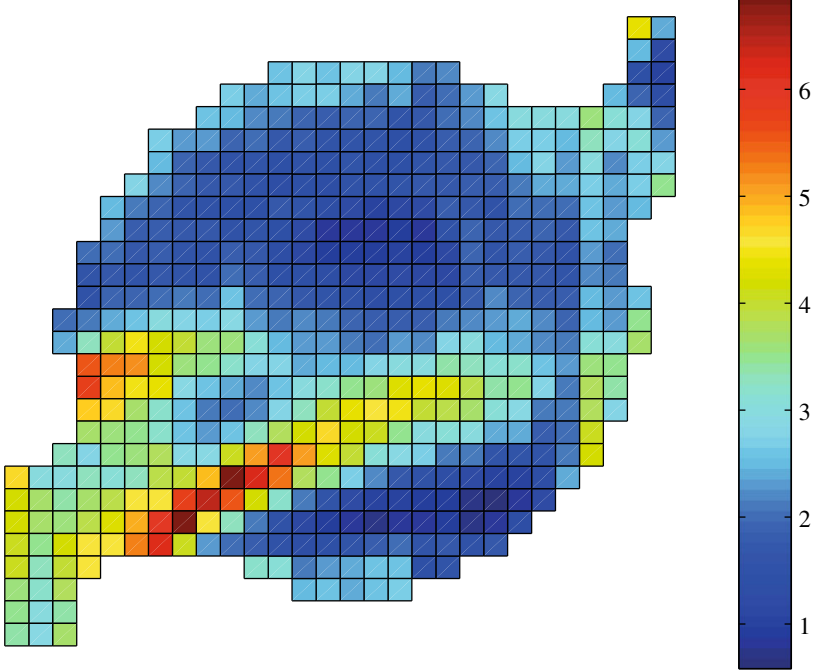

Fig. 14 Local values of the dissipation rate for the experiment with salt solution in the uncoated large structure at $R e_{p}=457$

estimated values are lower than the real ones because small-scale velocity gradients are filtered. The discussion of the effect of measurement resolution is found in many publications (Saarenrinne and Piirto 2000; Saarenrinne et al. 2001; Baldi and Yianneskis 2003; Delafosse et al. 2011; Sharp and Adrian 2001). These publications illustrate the inherent uncertainties of PIV for dissipation rate measurements. A further challenge is that the dissipation rate is not homogeneous, and therefore, there are regions in the porous structure where the Kolmogorov length scale is even smaller. A local distribution of measured dissipation rate values is shown in Fig. 14.

The region of maximum dissipation rate is found at the boundary of the jet entering the cell. Local values of the dissipation rate are more than 2.5 times higher than the average. Therefore, according to Eq. (22), the Kolmogorov length scale in these regions would be $20 \%$ lower than the average. In these regions, the error from insufficient resolution would be even higher. The most important challenge regarding resolution is, however, that the true value of the dissipation rate is unknown. $l_{K}$ was determined using the measured dissipation rate which is known to be underestimated. In the following, we discuss the error propagation in the estimation of the Kolmogorov length scale. According to Saarenrinne et al. (2001), a resolution of $\Delta=$ $9 l_{K}$ leads to a dissipation rate that corresponds to $65 \%$ of the true value. Assuming $\Delta=9 l_{K}$, we could therefore recalculate the Kolmogorov length scale with a corrected dissipation rate of $\hat{\varepsilon}=1 / 0.65 \cdot \varepsilon$. With this assumption, we obtain $\hat{l_{K, \text { min }}}=40 \mu \mathrm{m}$ and $\hat{l_{K, \text { max }}}=88 \mu \mathrm{m}$. This corresponds to a $10 \%$ decrease. This means that large changes in the dissipation rate only lead to small changes in the Kolmogorov length scale. Even if the dissipation rate was 


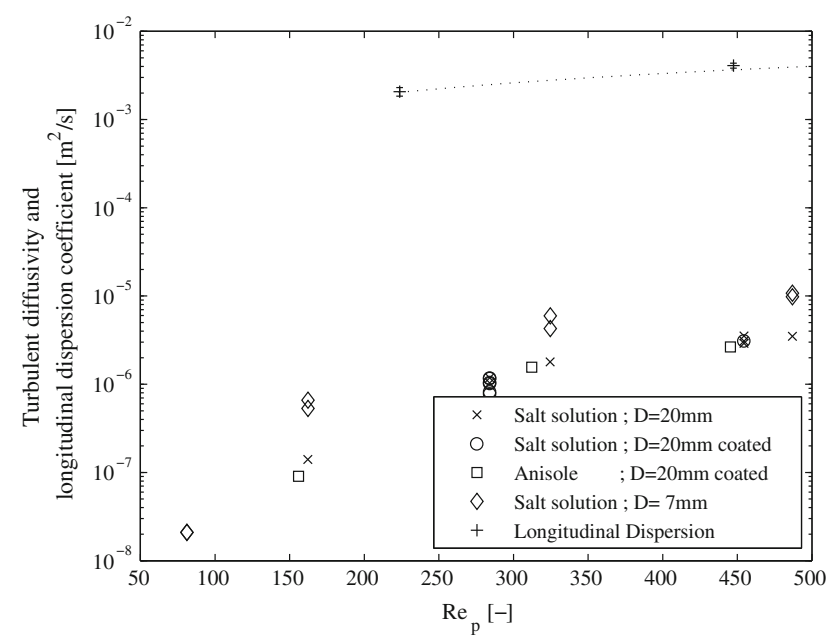

Fig. 15 Comparison of turbulent diffusivity measured by PIV to longitudinal dispersion coefficient determined by pulse experiments (Häfeli et al. 2013)

ten times higher, this would only result in a $44 \%$ smaller Kolmogorov length scale. The relative resolution would then be 4.8-10. With this resolution, the estimated values would still have a reasonably low error. From this, we conclude that $\Delta / l_{K}$ is not much larger than the above estimated 2.7-6.

\subsection{Turbulent diffusivity}

Finally, we want to use the measured quantities to investigate mass transport phenomena. In a previous study (Häfeli et al. 2013), the axial dispersion in a flow through the porous structure was investigated. Dispersion of mass in axial direction is governed by three effects: molecular diffusion, Taylor dispersion and turbulent mixing. By pulse experiments, the dispersion coefficient is obtained that includes all of these effects. The relative contribution of different mechanisms remains, however, unknown. We therefore want to use the PIV measurements to estimate the contribution of turbulent mixing to axial dispersion. This is done by employing the $k-\varepsilon$ model introduced in Sect. 2.1. The resulting turbulent diffusivity is shown as a function of pore Reynolds number in Fig. 15. We observe that the turbulent diffusivity is a strong function of the pore Reynolds number. Overall, it increases by more than an order of magnitude when the Reynolds number is increased from 150 to 500. This is expected as the measurements cover a wide range from unsteady laminar up to fully turbulent flow. However, if we compare the values of the turbulent diffusivity to the longitudinal dispersion, we find that the values are three orders of magnitude lower even at the highest Reynolds number. Recalling that the dissipation rate is underestimated, we expect that the real turbulent diffusivity is even smaller (see Eq. 5). The longitudinal dispersion is therefore dominated by other effects. The molecular diffusion $\left(D_{m}=1.9 \mathrm{e}-9 \mathrm{~m}^{2} / \mathrm{s}\right)$ can be excluded as it is even smaller than the turbulent diffusivity. We therefore conclude that the axial dispersion at the investigated Reynolds numbers is mainly governed by Taylor dispersion.

The transition from Taylor dispersion to turbulent dispersion is also discussed by Hlushkou and Tallarek (2006). They note that the transition from the regime dominated by molecular diffusion to the regime of Taylor dispersion is usually defined by the Peclet number. Many authors also used the Peclet number to define the transition from Taylor dispersion to turbulent dispersion. This was questioned by Hlushkou and Tallarek (2006) as the molecular diffusion is involved neither in Taylor dispersion nor in turbulent transport, and therefore, the transition should be rather described by means of a Reynolds number. By estimating the Reynolds numbers of previous studies, they found that the transition occurs at a superficial Reynolds number corresponding to the onset of fluctuations. In our study, we observed a steep increase in turbulent diffusivity in the range between unsteady laminar and turbulent flow. However, at the investigated Reynolds numbers, the Taylor dispersion still outweighs the turbulent transport.

\section{Conclusions}

An aqueous solution of $\mathrm{NaI}$ and $\mathrm{ZnI}_{2}$ is introduced as a new index-matching fluid for optical measurements. This enables PIV measurements inside a designed porous structure manufactured by rapid prototyping. Due to oxidation, the salt solution changed its color from transparent to yellow. It was shown that a Nd:YAG laser of $532 \mathrm{~nm}$ is not affected by the light absorption which occurs in the blue region. It was further shown that the absorption of water in the structure could successfully be reversed by flushing the structure with dry air or by exposure to vacuum. Experiments with the newly introduced salt solution and with anisole were then made at two geometrical scales. By applying the appropriate scaling laws, we observed a good agreement of the measurements of turbulent kinetic energy and dissipation rate. The measurements were further used to discuss the local distribution of turbulent kinetic energy and dissipation rate. For the dissipation rate, different models were evaluated by considering the isotropy ratios. It was found that the ratios observed by Delafosse et al. (2011) are well in agreement with our values. Generally, the dissipation rate of turbulent kinetic energy is a quantity which is only accurately measurable if the flow field is resolved down to the Kolmogorov length scales. Usually, this is not possible in PIV experiments, and therefore, the measured dissipation rate is underestimated. 
In relation to the mean Kolmogorov length scale, the resolution was 2.7-6 times higher what means that the resolution is almost sufficient to accurately measure the dissipation rate. From the dissipation rate and turbulent kinetic energy, the turbulent diffusivity was estimated using the standard $k-\varepsilon$ model. We observe a steep increase in turbulent diffusivity when going from the unsteady laminar to the turbulent regime. However, the turbulent diffusivity makes up only a small part of the axial dispersion coefficient. Therefore, the axial dispersion in our structure at $R e_{p}=500$ is still mainly governed by Taylor dispersion.

Acknowledgments We gratefully acknowledge financial support by the Swiss National Science Foundation (SNF) under Grant Number 200021132552.

\section{References}

Baldi S, Yianneskis M (2003) On the direct measurement of turbulence energy dissipation in stirred vessels with PIV. Ind Eng Chem Res 42:7006-7016

Banhart J (2001) Manufacture, characterisation and application of cellular metals and metal foams. Prog Mater Sci 46:559-632

Budwig R (1994) Refractive index matching methods for liquid flow investigations. Exp Fluids 17:350-355

Butscher D, Hutter C, Kuhn S, Rudolf von Rohr Ph (2012) Particle image velocimetry in a foam-like porous structure using refractive index matching: a method to characterize the hydrodynamic performance of porous structures. Exp Fluids 53:1123-1132

Delafosse A, Collignon M-L, Crine M, Toye D (2011) Estimation of the turbulent kinetic energy dissipation rate from 2D-PIV measurements in a vessel stirred by an axial Mixel TTP impeller. Chem Eng Sci 66:1728-1737

de Jong J, Cao L, Woodward SH, Salazar JPLC, Collins LR, Meng H (2009) Dissipation rate estimation from PIV in zero-mean isotropic turbulence. Exp Fluids 46:499-515

Doron P, Bertuccioli L, Katz J, Osborn TR (2000) Turbulence characteristics and dissipation estimates in the coastal ocean bottom boundary layer from PIV data. J Phys Oceanogr 31:2108-2134

Dukhan N, Suleiman AS (2014) Simulation of entry-region flow in open-cell metal foam and experimental validation. Transp Porous Media 101:229-246

Dybbs A, Edwards RV (1984) A new look at porous media fluid mechanics-Darcy to turbulent. In: Bear J, Corapcioglu MY (eds) Fundamentals of transport phenomena in porous media, NATO ASI Series E, vol 82. Martinus Nijhoff, Dordrecht, pp 199-256

Efron B (1979) Bootstrap methods: another look at the jackknife. Ann Stat 7:1-26

Gabriele A, Nienow AW, Simmons MJH (2009) Use of angle resolved PIV to estimate local specific energy dissipation rates for up- and down-pumping pitched blade agitators in a stirred tank. Chem Eng Sci 64:126-143

George WK, Hussein HJ (1991) Locally axisymmetric turbulence. J Fluid Mech 233:1-23

Häfeli R, Hutter C, Damsohn M, Prasser H-M, Rudolf von Rohr Ph (2013) Dispersion in fully developed flow through regular porous structures: experiments with wire-mesh sensors. Chem Eng Sci 69:104-111

Hassan YA, Dominguez-Ontiveros EE (2008) Flow visualization in a pebble bed reactor experiment using PIV and refractive index matching techniques. Nucl Eng Des 238:3080-3085

Hlushkou D, Tallarek U (2006) Transition from creeping via viscousinertial to turbulent flow in fixed beds. J Chromatogr A 1126:70-85

Horneber T, Rauh C, Delgado A (2012) Fluid dynamic characterisation of porous solids in catalytic fixed-bed reactors. Microporous Mesoporous Mater. 154:170-174

Hutter C, Zenklusen A, Lang R, Rudolf von Rohr Ph (2011) Axial dispersion in metal foams and streamwise-periodic porous media. Chem Eng Sci 66:1132-1141

Hutter C, Zenklusen A, Kuhn S, Rudolf von Rohr Ph (2011) Large eddy simulation of flow through a streamwise-periodic structure. Chem Eng Sci 66:519-529

Jolls KR, Hanratty TJ (1966) Transition to turbulence for flow through a dumped bed of spheres. Chem Eng Sci 21:1185-1190

Jones WP, Launder BE (1972) The prediction of laminarization with a two-equation model of turbulence. Int $\mathrm{J}$ Heat Mass Transf 15:301-314

Launder BE, Sharma BI (1974) Application of the energy-dissipation model of turbulence to the calculation of flow near a spinning disc. Int J Heat Mass Transf 1:131-138

Melling A (1997) Tracer particles and seeding for particle image velocimetry. Meas Sci Technol 8:1406-1416

Narrow TL, Yoda M, Abdel-Khalik SI (2000) A simple model for the refractive index of sodium iodide aqueous solutions. Exp Fluids $28: 282-283$

Pope SB (2000) Turbulent flows. Cambridge University Press, Cambridge

Saarenrinne P, Piirto M, Eloranta H (2001) Experiences of turbulence measurements with PIV. Meas Sci Technol 12:1904-1910

Saarenrinne P, Piirto M (2000) Turbulent kinetic energy dissipation rate estimation from PIV velocity vector fields. Exp Fluids 29(Suppl.):S300-S307

Sharp KV, Adrian R (2001) PIV study of small-scale flow structures around a Rushton turbine. AIChE J 47(4):766-778

Somos DSM (2012) Product Data Somos WaterShed XC11122. www. $\mathrm{dsm} . \mathrm{com} / \mathrm{content} / \mathrm{dam} / \mathrm{dsm} /$ somos/en_US/documents/somoswatershed-xc-11122-datasheet (accessed 23rd Oct 2013)

Wiederseiner S, Andreini N, Epely-Chauvin G, Ancey C (2011) Refractive-index and density matching in concentrated particle suspensions: a review. Exp Fluids 50:1183-1206

$\mathrm{Xu}$ D, Chen J (2013) Accurate estimate of turbulent dissipation rate using PIV data. Exp Therm Fluid Sci 44:662-672

Zenklusen A, Kenjeres S, Rudolf von Rohr Ph (2014) Vortex shedding in a highly porous structure. Chem Eng Sci 106:253-263 